\title{
The study of power transformer differential protection's operation in the internal fault conditions
}

\author{
Ilya Litvinov ${ }^{1, *}$, Aleksandra Naumova $^{1}$, Vasiliy Titov ${ }^{1, *}$, Andrey Trofimov $^{1}$, and Elena Gracheva ${ }^{2}$ \\ ${ }^{1}$ Novosibirsk State Technical University, Novosibirsk, Russia \\ ${ }^{2}$ Kazan State Power Engineering University, Kazan, Russia
}

\begin{abstract}
Special attention is paid to high-speed relay protections' operation in transient modes due to a number of major failure events that have occurred over the past 10 years in the power system of the Russian Federation. Operation of power transformer's differential protection in case of internal short circuit is studied in this research. False blocking of protection is possible in such mode due to saturation of current transformers. A value of blocking time may exceed the maximum permissible short-circuit disconnection time under conditions of maintaining the dynamic stability of the power system. Primary and secondary currents in transient modes are obtained by simulation of short circuits. Windings of the modeled current transformers are connected in a star to a null wire. RMS values are calculated using a mathematical model of the Fourier filter. The current transformers were checked according to the methods declared in PNST 283-2018 and GOST R 58669-2019. The analysis carried out in this work allows to estimate possibility of long-term blocking of the differential protection of a power transformer in case of internal short circuit, especially in case of significant value of time constants.
\end{abstract}

\section{Introduction}

The problem of relay protection's stable operation in case of deep saturation of current transformers (CT) is relevant in Russian Federation [1-4]; it has obtained exceptional topicality since 2014 [5-7]. First of all, it is caused by widespread using of CT, having accuracy class 10P and absence of regulations of relay protection's operation during transient processes. It is worth clarifying that the problem is relevant in some other countries, such as China [8-10], India [11-13], Romania [14,15], and Brazil [16,17].

Regulations are still absent on CT with accuracy classes PR, TPY, TPZ. Moreover, replacement of CT takes a long time and significant resources. Existing standards PNST 283-2018 and GOST R 58669-2019 [18, 19], that allow calculate saturation time, often overstate requirements to $\mathrm{CT}$ and relay protection. So, estimation of relay protection will be relevant for a long time in Russian Federation.

In this work, such disoperation of protection is studied using the example of differential protection (DP) of a power transformer (PT). Such protection may be blocked if there is special programming against incorrect operations in case of magnetizing inrush based on detection of second harmonic of fault current. Such programming is applied in modern micro processional terminals $[20,21,22]$.

Let us take a closer look of a case an internal fault, when a CT is deeply saturated. The secondary current is distorted (Fig. 1).

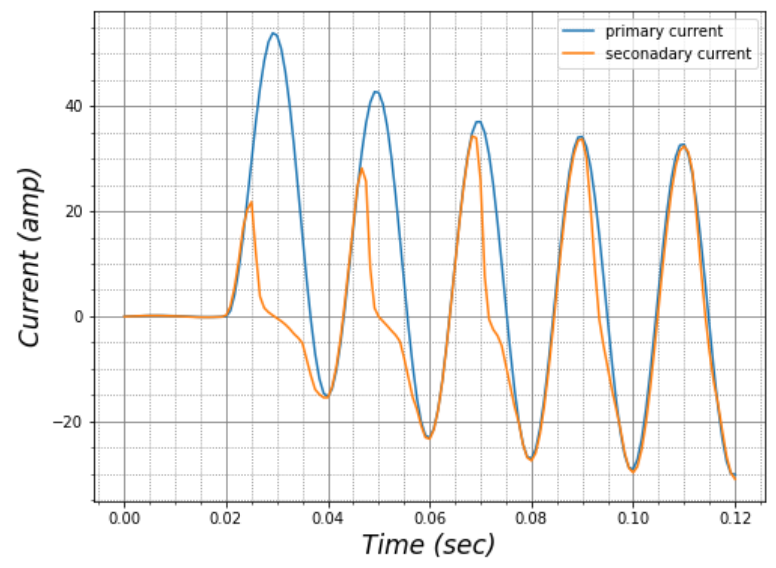

Fig. 1. Currents' oscillogram.

The primary current is shown by the blue line, distorted secondary current - by the orange one.

The value of magnetizing inrush is similar to the value of a short-circuit current, but it is not a fault and it does not require tripping, so the protection have to be blocked.

However, there is significant content of the second harmonic in distorted secondary current (Fig. 1, orange line) leads to disoperation of the protection until the CT desaturates, because the regime is recognized as a magnetizing inrush [23]. So, the time of the relay protection's operation is significantly increased. Such delay lead to the following consequences:

- high-priced power transformer's repair

\footnotetext{
* Corresponding author: vtitovnstu@gmail.com
} 
- dynamic stability upset and electric power supply interruption as the result

- cascaded development of a fault in case of existing of other derangements that lead to considerable diseconomies to electric energy system and load customers

The consequences show that such delay has to be considered as a disoperation. That is why, estimation of probability of differential protection's operation in external short circuit mode is the aim of this scientific work.

The following steps are to be made:

- creation of required models;

- definition of parameters, influencing on the value of relative fraction of the second harmonic in the secondary current;

- definition of experiments' circumstances

- analysis of the results

\section{Used models}

\subsection{The model of primary network}

Data collection is made with the help of simulation of big number of short circuits in such student versions of software as ARM SRZA and Matlab Simulink.

An equivalent network of a real part of electrical network and its parameters in ARM SRZA is shown in the Fig. 2.

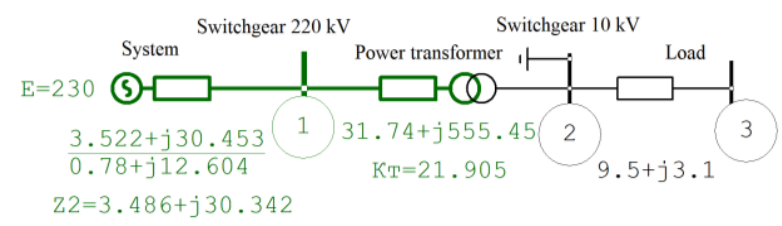

Fig. 2. An equivalent network in ARM SRZA.

An investigated CT is set at the high-voltage (HV) side of a PT. In-feeding of a short circuit in case of internal fault is only from the HV-side, so, stability of PT's DP operation fully depends on CT's saturation at the HV-side of the PT. This CT is side-mounted; bus system is in the DP's zone of operation.

In case of three-phase short circuit in the point 1 the primary current of the $\mathrm{CT}$ is $4332 \mathrm{~A}$. The most significant in-feeding of a short circuit from a power system is in case of one-phase short-circuit - 5298 A. One-phase short circuit has the largest probability in 220 $\mathrm{kV}$ networks [24], so, they will be studied further in this work.

A model in Simulink is used for transient processes simulating (Fig. 3).

Parameters of unit "System" (it is modeling the power system) are set in order to the definite value of three-phase short circuit current (5298 A) for simplification of one-phase short circuit modelling. The place of a short circuit is defined with help of the unit "Fault". Parameters of the unit "System" are following: the rated voltage $\underline{E}=230 \angle 0^{\circ} \mathrm{kV}$; inductivity $L=$ $0,079 H$; active resistance $R=2,651 \Omega$.
Parameters of unit «Power_transformer» are defined by the resistance of a PT: inductivity $L=1,765 \mathrm{H}$; active resistance $R=31,74 \Omega$.

The unit «Load220» models power load: $\underline{S}=9,5+$ j3,1 MVA.

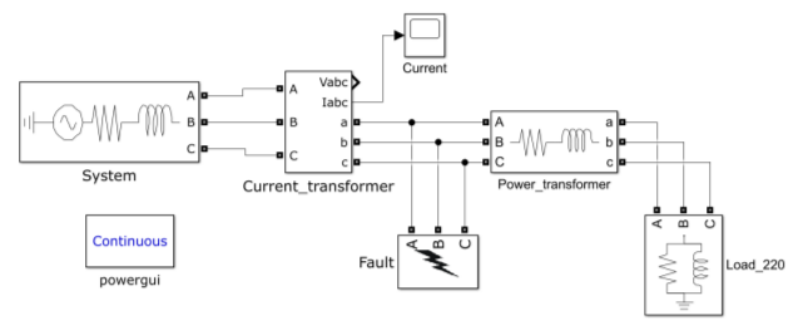

Fig. 3. An equivalent network in Simulink.

\subsection{The model of saturating current transformer}

One of possible CT's models is used in this work. Equivalent network of a saturating $\mathrm{CT}$ is shown below (Fig. 4).

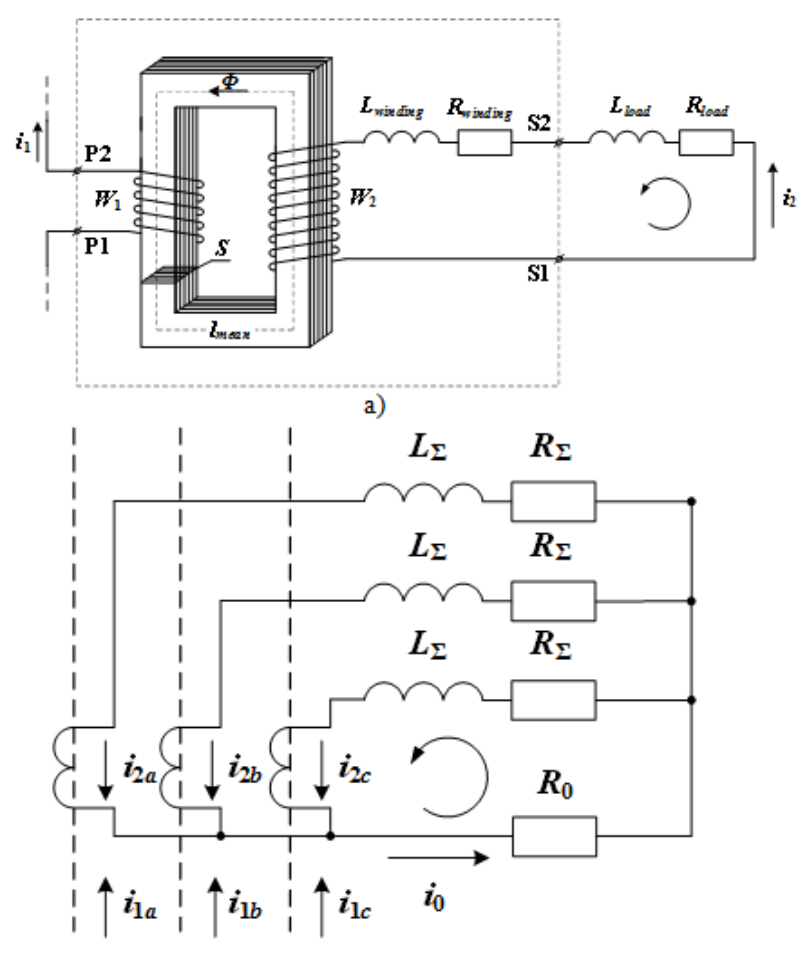

b)

Fig. 4. Equivalent network of a saturating CT: a) - a single CT; b) - in a star to a null wire.

Such models (Fig. 4) are described by these systems of equations:

$$
\left\{\begin{array}{c}
\frac{d i_{2}}{d t} L_{\Sigma}+i_{2} R_{\Sigma}=\frac{d B}{d t} S W_{2} \\
\frac{d B}{d t}=\mu_{\partial} \frac{\frac{d i_{1}}{d t} W_{1}-\frac{d i_{2}}{d t} W_{2}}{l_{\text {mean }}}
\end{array}\right.
$$




$$
\left\{\begin{array}{c}
\frac{d i_{2 a}}{d t} L_{\Sigma}+i_{2 a} R_{\Sigma}+i_{0} R_{0}=\frac{d B_{a}}{d t} S W_{2} \\
\frac{d i_{2 b}}{d t} L_{\Sigma}+i_{2 b} R_{\Sigma}+i_{0} R_{0}=\frac{d B_{b}}{d t} S W_{2} \\
\frac{d i_{2 c}}{d t} L_{\Sigma}+i_{2 c} R_{\Sigma}+i_{0} R_{0}=\frac{d B_{c}}{d t} S W_{2} \\
\frac{d B_{a}}{d t}=\mu_{d a} \frac{\frac{d i_{1} a}{d t} W_{1}-\frac{d i_{2 a}}{d t} W_{2}}{l_{\text {mean }}} \\
\frac{d B_{b}}{d t}=\mu_{d b} \frac{\frac{d i_{1 b}}{d t} W_{1}-\frac{d i_{2 b}}{d t} W_{2}}{l_{\text {mean }}} \\
\frac{d B_{c}}{d t}=\mu_{d c} \frac{\frac{d i_{1 c} c}{d t} W_{1}-\frac{d i_{2 c}}{d t} W_{2}}{l_{\text {mean }}}
\end{array}\right.
$$

where:

$i_{2 k}-$ instantaneous secondary current in phase $k, \mathrm{~A}$;

$i_{1 k}-$ instantaneous primary current in phase $k, \mathrm{~A}$;

$t$ - time, $\mathrm{s}$;

$L_{\Sigma}=L_{\text {winding }}+L_{\text {load }}-$ total inductivity of secondary circuit, $\mathrm{H}$;

$R_{\Sigma}=R_{\text {winding }}+R_{\text {load }}-$ total active resistance of secondary circuit, $\Omega$;

$B_{k}$ - magnetic induction in a magnetic core of a CT in phase $k, \mathrm{~T}$;

$S$ - a core cross-sectional area, $\mathrm{m}^{2}$;

$W_{2}$ - number of rounds of secondary winding;

$W_{1}$ - number of rounds of primary winding;

$\mu_{d k}-$ differential magnetic permeability in $\mathrm{CT}$ in phase $k, T \cdot m / A$;

$l_{\text {mean }}$ - length of mean magnetic line, $\mathrm{m}$;

$i_{0}=i_{2 a}+i_{2 b}+i_{2 c}-$ current in a zero wire;

$R_{0}$ - zero wire resistance.

There are parameters of chosen CT and the results of it's testing for estimated error and time of saturation in tables $1-2$.

It is possible to use the described model in the research for these reasons:

- coincidence of transient process nature in (Fig. 1, orange line) with published in [25];

- coincidence of calculated saturation time with the moment of current skip (Fig. 1);

- in case of nominal relative short-circuit current and nominal load the error in a steady-state regime of a short circuit is about $10 \%$.

Parameters of CT a taken from [25] and it is possible, that the magnetic core is made from steel E330A. Windings of the current transformers are connected in a star to a null wire, because the phase shift is eliminated algorithmically.

Saturation time calculation is made by formulas from addendum page B [18]. It is set that time, necessary for precise measurement is $25 \mathrm{~ms}$ [20]. The chosen CT does not meet requirements [18] in case of residual magnetic induction and it is necessary to use a CT with better characteristics or with larger primary current or with nonmagnetic gap (10PR, for example).

However, the standard [18] has become valid only since 2018. Before that, current transformers were chosen without calculation of saturation time. Therefore, it is possible to study the chosen CT. Nominal secondary current of the CT is $5 \mathrm{~A}$ to demonstrate that small saturation time does not warrant of protection's wrong disoperation.

As a result, differential protection of power transformers with single power supply on microprocessor-based terminals, powered from current transformers (class of precision 10P) is in the focus of this research.

\subsection{The model of a Fourier filter}

Modern microprocessor-based terminals of relay protection use effective current for estimation of relative fraction of the second harmonic in secondary current. A Fourier filter is the simplest and the most precise device for it. It's model is described by the following equation [26]:

$$
\underline{F}(n T)=\frac{2 j}{N} \sum_{n-N+1}^{n} u(n T) e^{-j \omega_{0} n T}
$$

where:

$F(n T)$ - a value of filter's input signal at the moment of time $n T$ ( $n-\mathrm{a}$ whole number);

$u(n T)$ - a value of filter's output signal at the moment of time $n T$;

$T=1 / 1200 s-$ sampling period $(1200 \mathrm{~Hz}$ for modern microprocessor-based devices);

$N=0,02 \cdot 1200=24 \quad-$ a number of sample captures during the period of the main frequency;

$\omega_{0}=2 \cdot \pi \cdot 50=314 \mathrm{rad} / \mathrm{s}$ - the main angular rate.

\section{Significant parameters for the value of relative fraction of the second harmonic in secondary current}

The relative fraction of the second harmonic in secondary current is defined by the formula:

$$
\Delta=\frac{I_{(2)}}{I_{(1)}} \cdot 100 \%
$$

where:

$I_{(2)}$ - an effective value of the second harmonic, A;

$I_{(1)}$ - an effective value of the main harmonic, A.

If the value of $\Delta$ exceeds the operating value, the protection is blocked. The recommended operating value of the leading manufacturers ("Ekra", Siemens, GE) is from 10 to $15 \%$ [20-22].

The relative fraction of the second harmonic in secondary current depends on the following factors:

- parameters of a short circuit (relative short-circuit current, it's moment and place);

- a value CT core's residual magnetic induction;

- load value and it's type;

- aperiodic time constant in the primary network;

- the value of the transient resistance at the point of short circuit;

- circuit-mode parameters. 
Table 1. Parameters of TFND-220-I-HL.

\begin{tabular}{|c|c|c|c|c|c|c|c|c|c|c|}
\hline 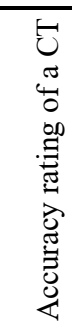 & 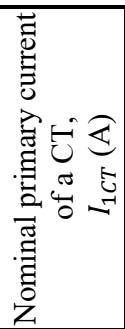 & 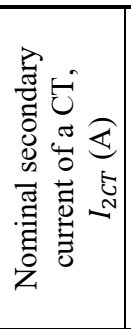 & 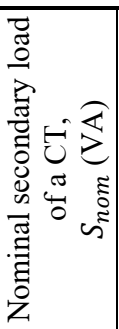 & 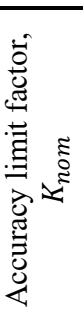 & 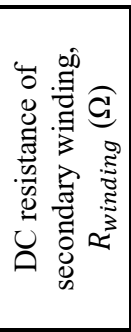 & 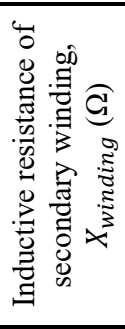 & 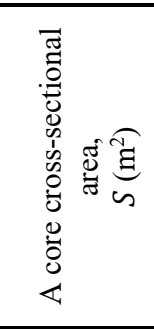 & 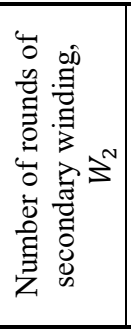 & 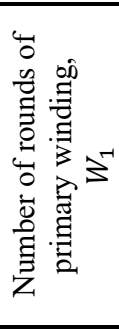 & 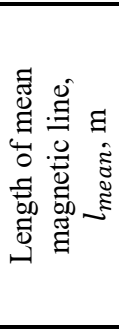 \\
\hline $10 \mathrm{P}$ & 1200 & 5 & 50 & 10 & 0,51 & 0,05 & $30,5 \times 10^{-4}$ & 240 & 1 & 1,55 \\
\hline
\end{tabular}

Table 2. Defining a value of the CT error $(<10 \%)$ and the current transformer's saturation time.

\begin{tabular}{|c|c|c|c|c|c|c|c|c|}
\hline Parameter & 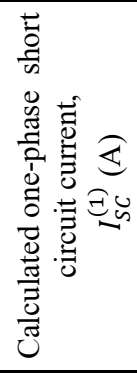 & 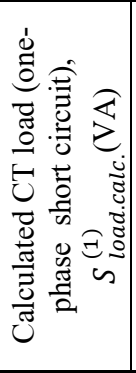 & 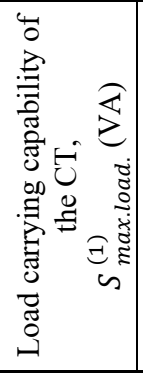 & 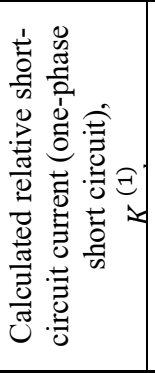 & 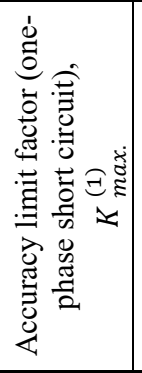 & 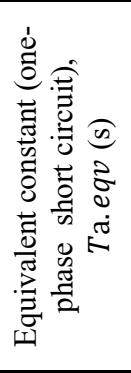 & 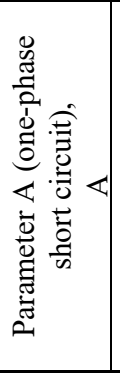 & 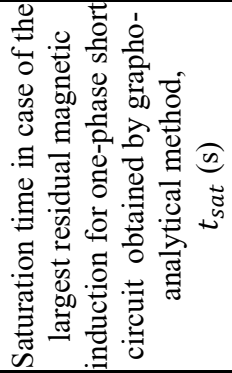 \\
\hline Value & 5298 & 92,8 & 129,5 & 4,4 & 6 & 0,03 & 1,316 & 0,005 \\
\hline
\end{tabular}

In order to simplify the modeling, the following assumptions are made:

a) circuit-mode parameters are constant

b) only solid one phase fault (phase A) are calculated (a transient resistance may be changed in wide range)

c) according to [25], an error of the CT during a transient process is extremely weakly dependent on the change in the secondary load by $1.5-2$ times (excepting the first half-period since the moment of short circuit beginning); by this reason the value of the load is constant and it is active.

\section{Experimental conditions}

\subsection{Initial data for modeling}

According to [27], the maximum permissible value of the short circuit breaking time is limited by the condition of dynamic stability (160 ms for $220 \mathrm{kV}$ network). Considering that the shutdown time of modern circuit breakers is about $80 \mathrm{~ms}, 80 \mathrm{~ms}$ also remains for the operation of a high-speed DP.

Since the Fourier filter calculates the effective value of the signal (the effective value is an integral parameter that can be determined only after one integration period, equal to one period of the industrial frequency), then in all experiments there is no short circuit in the system during the first $0.02 \mathrm{~s}$. Therefore, the simulation time increases by $20 \mathrm{~ms}-$ it is $100 \mathrm{~ms}$.

The moment of the short-circuit changes during one period; therefore the final simulation time is $120 \mathrm{~ms}$.

Despite the deep saturation of the CT, the effective value of the differential current will always be higher than the setting of protection, and therefore the failure in it's operation is determined only by blocking by second harmonic detection.

If the protection operates within $25 \mathrm{~ms}$ from the moment the short circuit's beginning, then such a trip of the protection is considered as normal.

If the protection operates no later than $80 \mathrm{~ms}$ after the start of the short circuit, then such a delay is considered acceptable, the stability of the EPS is not violated.

If the protection is triggered later, then such a delay is considered unacceptable, which is tantamount to a failure in operation, which means that violations of the dynamic stability of the EPS are possible.

It is assumed that the aperiodic component in the primary current (which depends on the short-circuit moment) and the residual induction in the core have the greatest influence on the CT saturation depth.

A short circuit occurs with an equal probability in the time interval from 0.02 to 0.039 seconds from the moment of the start of the simulation (there is no statistical data on the moments of short circuit, so, it was decided to take the same probability). The residual induction also varies in the range from -1.48 to $1.48 \mathrm{~T}$ with equal probability (which corresponds to $86 \%$ of the saturation induction of the steel of the selected grade $1.72 \mathrm{~T})$. The total number of experiments is 200 .

It is worth clarifying that the article considered the influence of the second harmonic braking organ on the operation of the DP sensitive organ without taking into account the differential cutoff, which is not blocked by the second harmonic braking. The short circuit mode on the HV side was also considered when the protection on was turned on the side-mounted CT. 


\subsection{Experiments' setting operations}

1) Arrays of 20 values of residual magnetic induction and 10 values of short-circuit moments are formed. The probability of each value appearing is the same.

2) Further single-phase short circuits are modeled for each moment of short circuit at all values of residual magnetic induction. After simulation, we get an array of 200 oscillograms of secondary currents. An example of such an oscillogram is in Figure 1.1 (orange curve).

3) According to formula 2.3, the effective values of the main and the second harmonics are calculated (Fig. 5).

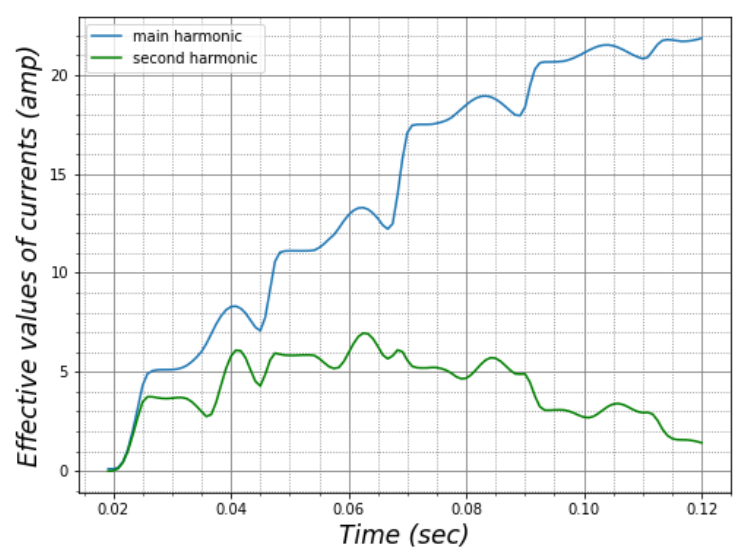

Fig. 5. Effective values of currents.

4) The relative fraction of the second harmonic in the differential current is determined according to formula (4), (Fig. 6). The moment of time, until that the protection's operation is not delayed is shown by dotted orange line. The moment of time, until that delay of protection's operation is acceptable is shown by dotted red line. Protection operation to the right from the dotted red line is considered unacceptable, because violation of EPS stability is possible in this case.

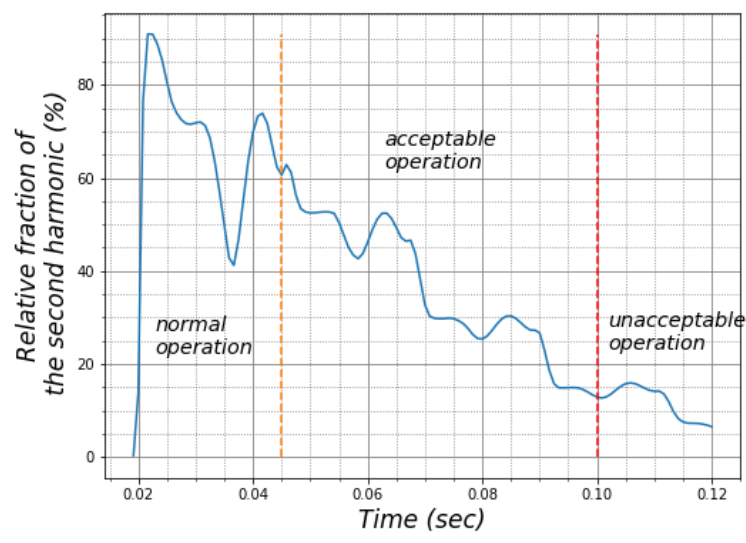

Fig. 6. Relative fraction of the second harmonic.

For each sample, the nature of the protection operation is determined ("normal operation", "admissible delay", "unacceptable delay"). Experiments are made with different second harmonic blocking settings.

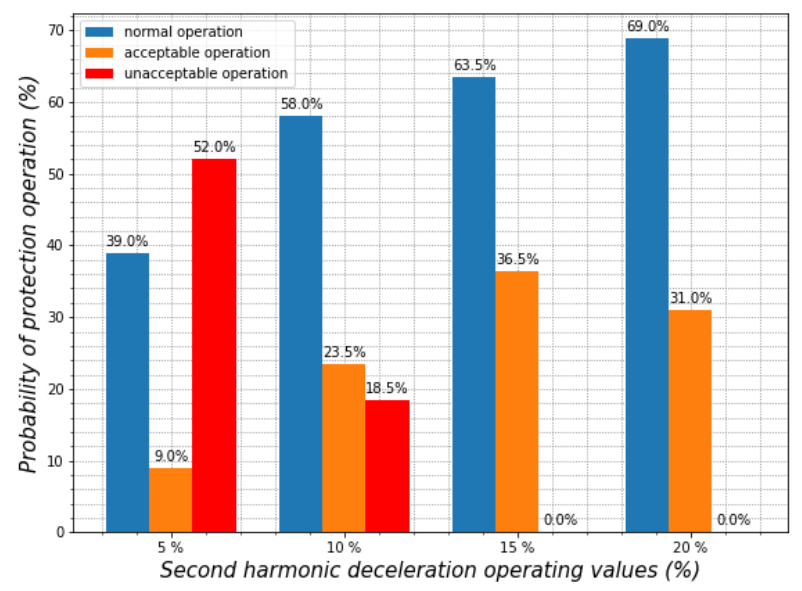

Fig. 7. Probability of protection operation.

These probabilities were determined for various second harmonic deceleration operating values.

The following conclusions can be made according to Fig. 7.

1) If the blocking setting's value is $10 \%$, the protection is operates without delay in $58 \%$ of cases, if this setting is $15 \%$ - in almost $64 \%$ of cases.

2) If the blocking setting's value is $10 \%$, then in almost $19 \%$ of cases there is a failure in the operation of protection and a violation of the dynamic stability of the EPS is possible.

3 ) If the blocking setting's value is $15 \%$, there are no failures, but in this case, the relative fraction of timedelayed operations increases.

However, it should be noted here that the time constant in the studied network is 0.03 seconds. In case of a short circuit near power plants, the time constant can be significantly larger, therefore, the aperiodic component of the short circuit current will decay more slowly, which means that the CT will come out of saturation much later.

Fig. 8 illustrates the cases when the probability of a relay failure increases. The short-circuit currents are the same as in Fig. 7, but time constants have other values.

It should be noted that three-phase short circuits are the most dangerous to the EPS stability. Differential current in phase A will be determined as the difference between the currents of phases A and B. The transient process in these phases will proceed in different ways, because they are shifted by $120^{\circ}$ relative to each other.

Fig. 9 illustrates the cases of a three-phase short circuit with $T_{\text {a.eq. }} 0.03$ and $0.1 \mathrm{~s}$ for all possible combinations of short circuit moments (10 values in the interval from 0.02 to 0.039 seconds) and inductions of phases $\mathrm{A}, \mathrm{B}$ and $\mathrm{C}$ (10 values each in the range from 1.48 to $1.48 \mathrm{~T}$ ). The total number of experiments is 10000 for each $T_{\text {a.eq. }}$.

\section{Conclusion}

1) It is necessary to consider not only the value of its saturation time, but also it is necessary to pay attention to the logic of the operation of a particular protection making a choice of a CT. 

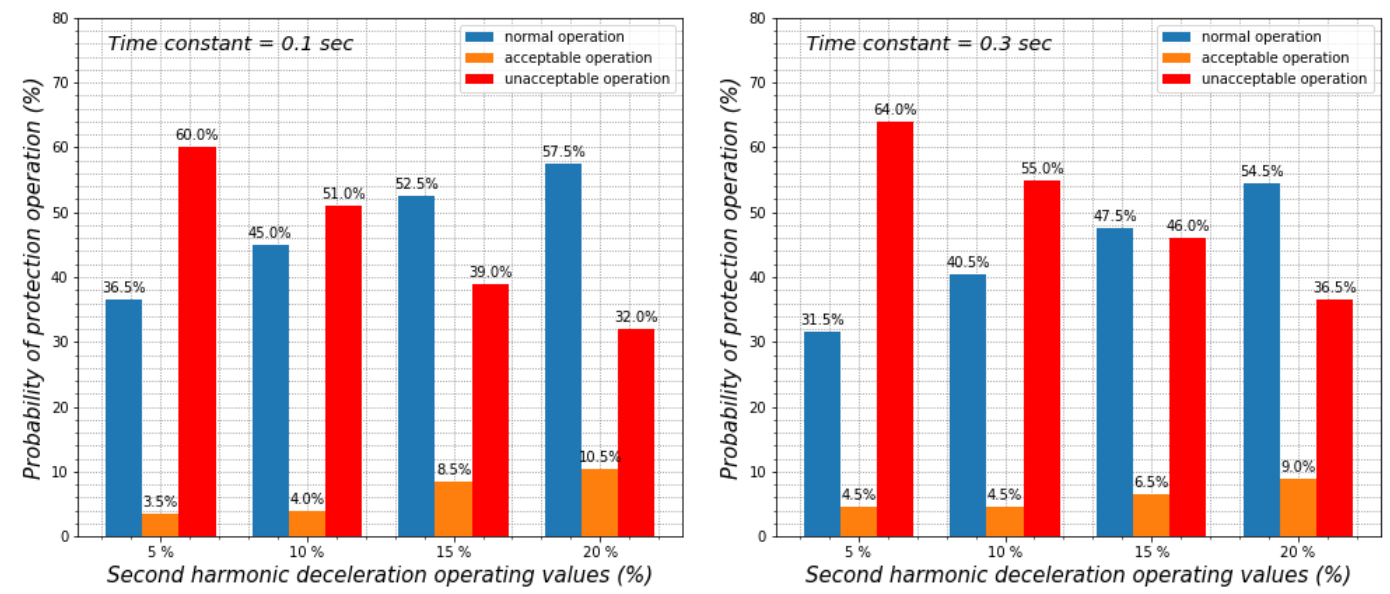

Fig. 8. Probability of protection operations in case of different values of $T_{\text {a.eq. }}$.
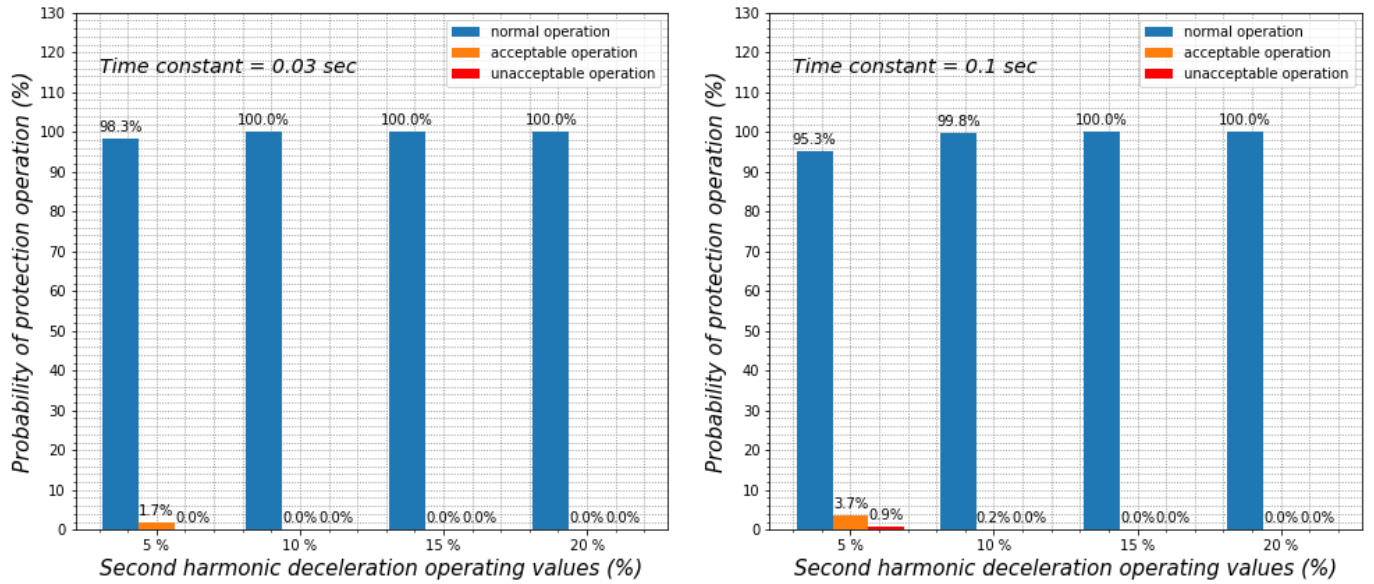

Fig. 9. Probability of protection operation in case of three-phase short circuit.

2) The analysis of the probability of the appearance of a relative fraction of the second harmonic makes it possible to justify the use of more expensive CTs. In the considered example, the time to saturation was $5 \mathrm{~ms}$, which indicated the need to use a CT with better characteristics. However, if the operation value of the second harmonic blocking is $15 \%$, the EPS instability does not occur.

3) The analysis of the probability of the appearance of the second harmonic relative fraction allows compare the possible risks of violation of the dynamic stability of the electric power system, as well as to justify the increase in capital investments to increase the reliability of the relay protection operation.

4) The analysis of the probability of occurrence of the second harmonic fraction helps to select the correct second harmonic blocking setting in terms of the possible risks of protection's operation failures. In the design calculations, the second harmonic blocking setting is assumed to be $10 \%$. Fig. 7 shows that with such an operation value, a violation of the dynamic stability of the EPS is probable, if the operation value is $15 \%$, there is no possibility of such a violation.

5) Due to blocking by the second harmonic, the reliability of the protection operation decreases at large values of the time constant (Fig. 8). This induces the need to use CT with accuracy classes PR, TPY, TPZ in order to reliably identify faults in electric power systems.

6) In case of a three-phase short circuit, stability of a power transformer differential protection's operation is higher than in case of one-phase short circuits.

This work was supported by Ministry of Education of the Novosibirsk Region (paper №2332 "The results of competitive selection for provision of targeted financial support to talented student youth - students of educational institutions of higher education located in the Novosibirsk Region, in 2020").

\section{References}

1. M. A. Kuparev, I. I. Litvinov and D. V. Baklanov, "Harmonic Analysis of the Currents in the Power Transformer Differential Protection Circuits in the Cases of External and Internal Faults," 2018 XIV International Scientific-Technical Conference on Actual Problems of Electronics Instrument Engineering (APEIE), Novosibirsk, Russia, 2018, pp. 202-209, doi: 10.1109/APEIE.2018.8545971.

2. Baklanov, Dmitry \& Litvinov, Ilya \& Kuparev, Mikhail \& Rusina, Anastasia. (2018). Power 
transformer adaptive differential cutoff. International Journal of Power and Energy Systems. 38. 10.2316/Journal.203.2018.1.203-0040

3. Litvinov, I.I., Glazyrin, V.E. Improved Algorithm for Phase Comparison for Differential Protection of a Power Transformer. Power Technol Eng 51, 251255 (2017). https://doi.org/10.1007/s10749-0170819-7

4. N. S. Buryanina, R. O. Gogolev, Y. F. Korolyuk, E. V. Lesnykh and K. V. Suslov, "Digital Differential Protection of the "Generator-Transformer» Block," 2019 International Science and Technology Conference "EastConf", Vladivostok, Russia, 2019, pp. 1-4, doi: 10.1109/EastConf.2019.8725341.

5. Press release "The system operator implements measures to prevent improper operation of relay protection devices" dated 12/14/2017 [Electronic resource]. - Access mode: http://so-ups.ru/ - Title from the screen. - (Date of request: 05/21/19).

6. Possibility of non-selective action of high-speed distance protection in case of external damage with high short-circuit currents / Doni N.A. - Relay, 2015, No. 04 (24), p. 30 - 33.

7. Degtyarev A.A., Kuzhekov S.L., Doni N.A., Shurupov A.A., Fedotov A.Yu. Analysis and verification of compliance of class $\mathrm{P}$ current transformers' characteristics with the operating conditions of the distance protection's first stages of power transmission lines during transient processes. Power stations. - 2020. - No. 3. - p. 43-53.

8. X. Wang, H. Liu, Z. Zhou, Y. Guo and D. Du, "A current transformer saturation identification method of transmission line based on current sample data," 2017 IEEE Power \& Energy Society General Meeting, Chicago, IL, 2017, pp. 1-6, doi: 10.1109/PESGM.2017.8274372.

9. T. Zheng, T. Huang, Y. Ma, Z. Zhang and L. Liu, "Histogram-Based Method to Avoid Maloperation of Transformer Differential Protection Due to Current-Transformer Saturation Under External Faults," in IEEE Transactions on Power Delivery, vol. 33, no. 2, pp. 610-619, April 2018, doi: 10.1109/TPWRD.2017.2712806.

10. Ma Jing, Xu Yan, Wang Zengping and Liu Haofang, "A novel adaptive scheme of discrimination between internal faults and inrush currents of transformer using mathematical morphology," 2006 IEEE Power Engineering Society General Meeting, Montreal, QC, Canada, 2006, pp. 7 pp.-, doi: 10.1109/PES.2006.1709011.

11. K. Kumar, G. B. Kumbhar and S. Mahajan, "A new efficient algorithm to detect Current Transformer saturation," 2016 IEEE Power and Energy Society General Meeting (PESGM), Boston, MA, USA, 2016, pp. 1-5, doi: 10.1109/PESGM.2016.7741583.

12. S. Murugan, S. P. Simon, S. Kinattingal, S. R. N. Panugothu and N. P. Padhy, "An empirical fourier transform based power transformer differential protection," 2017 IEEE Power \& Energy Society
General Meeting, Chicago, IL, 2017, pp. 1-1, doi: 10.1109/PESGM.2017.8274337.

13. H. S. Bhalja, B. R. Bhalja and P. Agarwal, "Rate of Rise of Differential Current Based Protection of Power Transformer," 2019 IEEE 16th India Council International Conference (INDICON), Rajkot, India, 2019, pp. 1-4, doi: 10.1109/INDICON47234.2019.9029009.

14. R. Radu, D. O. Micu, D. D. Micu and A. Ceclan, "Analysis of inrush and fault currents measurement errors generated by the current transformer saturation," 2014 49th International Universities Power Engineering Conference (UPEC), ClujNapoca, Romania, 2014, pp. 1-5, doi: 10.1109/UPEC.2014.6934699.

15. F. Mahfoud, G. C. Lazaroiu, T. Kherbek and T. Hayder, "Improvement of differential protection performance by external faults associated with a current transformer saturation," 2017 International Conference on ENERGY and ENVIRONMENT (CIEM), Bucharest, 2017, pp. 83-86, doi: 10.1109/CIEM.2017.8120812.

16. F. de Araujo Pereira, F. da Chagas Fernandes Guerra, E. Guedes da Costa and B. Alencar de Souza, "A Method for Correcting Distorted Current Waveforms at Air-Gapped Current Transformers," in IEEE Latin America Transactions, vol. 12, no. 4, pp. 596-601, June 2014, doi: 10.1109/TLA.2014.6868860.

17. F. Costa and R. Prado, "A Wavelet-Based Transformer Differential Protection With Differential Current Transformer Saturation and Cross-Country Fault Detection," 2018 IEEE Power \& Energy Society General Meeting (PESGM), Portland, OR, 2018, pp. 1-1, doi: 10.1109/PESGM.2018.8586618.

18. PNST 283-2018 Measuring transformers. Part 2. Specifications for current transformers (with Amendment).

19. GOST R 58669-2019 Unified power system and isolated power systems. Relay protection. Measuring inductive current transformers with closed magnetic circuit for protection. Guidelines for determining the time to saturation in case of a short circuit.

20. STO 56947007-29.120.70.99-2011 Guidelines for the selection of parameters for the operation of relay protection and automation devices of substation equipment manufactured by OOO NPP EKRA, JSC FGC UES.

21. STO 56947007-29.120.70.137-2012 Guidelines for selection of parameters of relay protection's operation and automation devices of the SIPROTEC (Siemens AG) series of transformers with a higher voltage of 110-220 kV, JSC FGC UES.

22. STO 56947007-29.120.70.109-2011 Guidelines for the selection of parameters for operation of relay protection and automation devices for substation 
equipment manufactured by GE Multilin, JSC FGC UES.

23. M. Jin and Y. Liu, "A new inrush current identification algorithm based on transformer core saturation," 2017 IEEE Power \& Energy Society General Meeting, Chicago, IL, 2017, pp. 1-5, doi: 10.1109/PESGM.2017.8274695.

24. Neklepaev BN Electrical part of power plants and substations: Textbook for universities. - 2nd ed., Rev. and add. - M.: Energoatomizdat, 1986. - 640 p.; with illustrations.

25. Korolev, E.P. Calculations of permissible loads in current circuits of relay protection / E.P. Korolev, E. M. Liberzon. - Moscow: Energy, 1980 - 208 p.

26. Shneerson E. M. Digital relay protection. - M.: Energoatomizdat, 2007 - 549 p.: with illustrations.

27. Guidelines for the sustainability of power systems. Approved by order of the Ministry of Energy of Russian Federation dated June 30, 2003 No. 277. 